\title{
Laser-Driven Proton Acceleration with Nanostructured Targets
}

Conference Paper · April 2017

DOI: $10.1117 / 12.2265913$

CITATIONS

6 authors, including:

Simon Vallières

Institut National de la Recherche Scientifique

14 PUBLICATIONS 106 CITATIONS

SEE PROFILE

Marianna Barberio

IPAT-LAB

87 PUBLICATIONS 417 CITATIONS

SEE PROFILE

Some of the authors of this publication are also working on these related projects:

Project Laser-Driven Proton Beams View project

Project Nanoparticle-Enhanced Radiation Therapy View project
READS

159

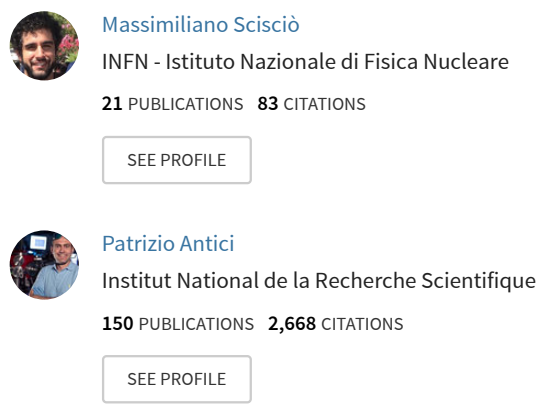




\section{Laser-driven proton acceleration with nanostructured targets}

Simon Vallières, Antonia Morabito, Simona Veltri, Massimiliano Scisciò, Marianna Barberio, et al.

Simon Vallières, Antonia Morabito, Simona Veltri, Massimiliano Scisciò, Marianna Barberio, Patrizio Antici, "Laser-driven proton acceleration with nanostructured targets," Proc. SPIE 10240, Laser Acceleration of Electrons, Protons, and lons IV, 1024009 (7 May 2017); doi: 10.1117/12.2265913

SPIE. Event: SPIE Optics + Optoelectronics, 2017, Prague, Czech Republic 


\title{
Laser-driven proton acceleration with nanostructured targets
}

\author{
Simon Vallières ${ }^{\mathrm{a}}$, Antonia Morabito ${ }^{\mathrm{b}, \mathrm{c}}$, Simona Veltri ${ }^{\mathrm{a}, \mathrm{b}}$, Massimiliano Scisciò ${ }^{\mathrm{a}, \mathrm{b}}$, Marianna \\ Barberio $^{\mathrm{b}, \mathrm{c}}$, and Patrizio Antici ${ }^{\mathrm{a}, \mathrm{b}}$ \\ ${ }^{a}$ INRS-EMT, 1650 blvd. Lionel-Boulet, Varennes (QC), Canada \\ bINFN-RM1 \& Uni. of Rome "La Sapienza", P. Aldo Moro, Rome, Italy \\ ${ }^{\mathrm{c} E L I-A L P S}$, ter 13 Dugonics, Szeged, Hungary
}

\begin{abstract}
Laser-driven particle acceleration has become a growing field of research, in particular for its numerous interesting applications. One of the most common proton acceleration mechanism that is obtained on typically available multi-hundred TW laser systems is based on the irradiation of thin solid metal foils by the intense laser, generating the proton acceleration on its rear target surface. The efficiency of this acceleration scheme strongly depends on the type of target used. Improving the acceleration mechanism, i.e. enhancing parameters such as maximum proton energy, laminarity, efficiency, monocromaticy, and number of accelerated particles, is heavily depending on the laser-to-target absorption, where obviously cheap and easy to implement targets are best candidates. In this work, we present nanostructured targets that are able to increase the absorption of light compared to what can be achieved with a classical solid (non-nanostructured) target and are produced with a method that is much simpler and cheaper than conventional lithographic processes. Several layers of gold nanoparticles were deposited on solid targets (aluminum, Mylar and multiwalled carbon nanotube buckypaper) and allow for an increased photon absorption. This ultimately permits to increase the laser-to-particle energy transfer, and thus to enhance the yield in proton production. Experimental characterization results on the nanostructured films are presented (UV-Vis spectroscopy and AFM), along with preliminary experimental proton spectra obtained at the JLF-TITAN laser facility at LLNL.
\end{abstract}

Keywords: nanostructured targets, nanoparticles, laser-matter interaction, laser-driven particle acceleration, target normal sheath acceleration, plasma physics

\section{INTRODUCTION}

Nanostructured surfaces have attracted a great deal of attention because of their excellent antireflection and light trapping effects. Patterning a smooth surface with nanopores or nanostructures has shown to reduce the surface reflection and hence to substantially increase the optical absorption in the visible range. ${ }^{1-3}$ Research on high absorption nanostructured materials is of crucial interest in many fields of material science since improvements on the material have a direct consequence on their applications. Therefore, in the last decades, strong effort has been put in the realization of nanostructured surface produced by superposition of a nanostructured layer onto a bulk surface or by the use of RX lithography. ${ }^{4-6}$ Many of these methods require very long and expensive processes without allowing a good control in the shape or dimensions of the realized nano-pattern. A critical point in the growth of nanostructured target is, in fact, the realization of a construct with a controlled topography and structure for minimizing the electrical resistance, increasing the optical absorption, and enhancing the mechanical properties, such as elasticity, plasticity and mechanical resistance. These properties are important for many applications such as optoelectronics, photocatalysis, green energy ${ }^{7}$ where an enhanced absorption results in increasing the device's performance and the overall operating efficiency. A more recent field of research where improved light absorption plays a crucial role is in laser-matter interactions with high-intensity lasers, in particular the field of laser-driven proton acceleration.

Further author information: (Send correspondence to S.V.)

S.V.: E-mail: simon.vallieres@emt.inrs.ca, Telephone: +1 5149638859

P.A.: E-mail: antici@emt.inrs.ca, Telephone: +1 5142286911

Laser Acceleration of Electrons, Protons, and Ions IV, edited by Eric Esarey, Carl B. Schroeder, Florian J. Grüner, Proc. of SPIE Vol. 10240, 1024009 - () 2017 SPIE · CCC code: 0277-786X/17/\$18 · doi: 10.1117/12.2265913 
One of the most common proton acceleration mechanisms that is obtained on typical multi-hundred TW laser systems is the so called Target-Normal-Sheath Acceleration (TNSA), ${ }^{8}$ in which protons are accelerated at the rear target surface of a solid foil (called target, typically made of $\mathrm{Au}, \mathrm{Al}$ or $\mathrm{Si}$ ), that is irradiated by a highintensity $\left(\mathrm{I}>10^{18} \mathrm{~W} / \mathrm{cm}^{2}\right)$, short pulse $(\mathrm{t}<1 \mathrm{ps})$ laser operating at central wavelengths around 800-1056 $\mathrm{nm}$. The laser-to-target absorption, i.e. how much energy is transferred from the laser to the target and from there to the particles, is a crucial parameter since it allows improving the efficiency of the acceleration mechanism. Currently this efficiency is at most in the order of a few percent and depends on the target and its structure. Several attempts have been made to use low-density, foam or micro- or nano-structured targets with the aim of improving the laser-to-target absorption..$^{9,10}$ These targets optimize the mean energy, collimation, and number of energetic "hot" electrons that are generated at the target front by the ponderomotive force, travel through the target bulk and establish at its rear surface the electrostatic field that drives the acceleration process. ${ }^{11}$ In this scenario, several groups ${ }^{12-15}$ demonstrated that the use of nanostructured targets could enhance the absorption of the laser pulse. However, most effort on these targets has been made trying to optimize the particle yield for the highest reachable energy range, while no consideration has been given for enhancing lower energy protons $(<3 \mathrm{MeV})$. These protons are particularly interesting for applications requiring a high flux of particles that deposit their energy within a short distance from the material surface e.g. warm dense matter, isochoric heating, astrophysics, nanostructure generation, micro- and nano-structure diagnostic etc.). They are also important for all applications where laser-driven proton acceleration shall replace classical low-energy proton beam generators, e.g. as used in material science diagnostic, ion spectroscopies, ion implantations, ion lithography, etc.).

In this work, we present nanostructured targets that are able to increase the absorption of light at least three times more than what can be achieved with a classical solid aluminum (non-nanostructured) target. We show that this absorption enhancement is very favorable for being applied in the laser-driven proton acceleration mechanism and in particular for enhancing the lower-energy proton yield. Compared to existing nanostructured targets that require the use of complex and expensive manufacturing procedures (e.g. lithographic methods) our targets present the advantage of being both, simple and cheap to manufacture, and do not exhibit the stringent laser-matter alignment conditions as required by microstructured targets. These targets are therefore a good candidate for being implemented on high-repetition-rate laser-driven beamlines where any efficiency enhancement in the laser-driven acceleration process is of high impact.

\section{FABRICATION OF NANOSTRUCTURED TARGETS}

In a recently published paper from our group ${ }^{16}$ an easy-to-use and inexpensive fabrication method to produce nanostructured targets is shown. Gold nanoparticles (GNPs) are first grown by laser ablation in solution (LASiS). A gold plate, with nominal purity greater than $99 \%$ (commercially available from Goodfellow) and dimensions of $1 \times 1 \mathrm{~cm}^{2}$, was placed on the bottom of a vessel cuvette containing an aqueous solution of acetone $(99.7 \%$ concentration; acetone is a very good solvent for the production of noble metal nanoparticles, as reported in previous studies $\left.{ }^{17}\right)$. The plate was ablated with the first harmonic $(1064 \mathrm{~nm}, 20 \mathrm{~Hz})$ of a pulsed Nd:YAG laser (7100 series of Quanta System). The laser spot covered the entire target surface with a fluence of 500 $\mathrm{mJ} /$ pulse. Using a controlled spray-dry process, the GNPs were then deposited on the surface of three different types of targets : aluminum foil (thickness of $15 \mu \mathrm{m}$ ), Mylar foil (thickness of $2.5 \mu \mathrm{m}$ ) and multiwalled carbon nanotube (MWCNT) buckypaper (thickness of $30 \mu \mathrm{m}$ ). The surface of the foils was heated in Ultra High Vacuum conditions in order to remove the adsorbed impurities prior to NP deposition.

The growth of NPs in solution during LASiS was characterized by measuring their extinction spectra with a typical UV-Vis spectrometer (TRIAX 320 from Horiba-Jobyn-Yvon). Further characterization to obtain morphological information was done through SEM and AFM microscopies. AFM images were obtained by the Icon AFM microscope from Bruker working in tapping mode. Each image was taken with a resolution of $512 \times 512$ pixels and a frequency of $1 \mathrm{~Hz}$. Dimensions of NPs, obtained from droplets deposited during LASiS on $\mathrm{SiO}_{2}$ surface, were analyzed by conducting a statistical analysis on 100 nanoparticles collected in several AFM images. For each sample, several areas (about 50) were scanned in windows of $500 \times 500 \mathrm{~nm}^{2}$ (resolution of $1024 \times 1024$ pixels). The images were then analyzed by the Nanoscope software (1.40 version from Bruker) to obtain morphological information and for measuring its dimensions. The radius of the nanoparticles was evaluated assuming that a spherical nanoparticle rests on the $\mathrm{SiO}_{2}$ surface and warps during the morphological analysis (in the 
interaction with the AFM tip) forming a spherical-cap-like structure. We evaluate the volume of each NP by a Bearing analysis and then estimate the radius assuming that the volume of spherical particle is conserved during the deposition and measuring processes. A few characterization results are presented on Figure 1.
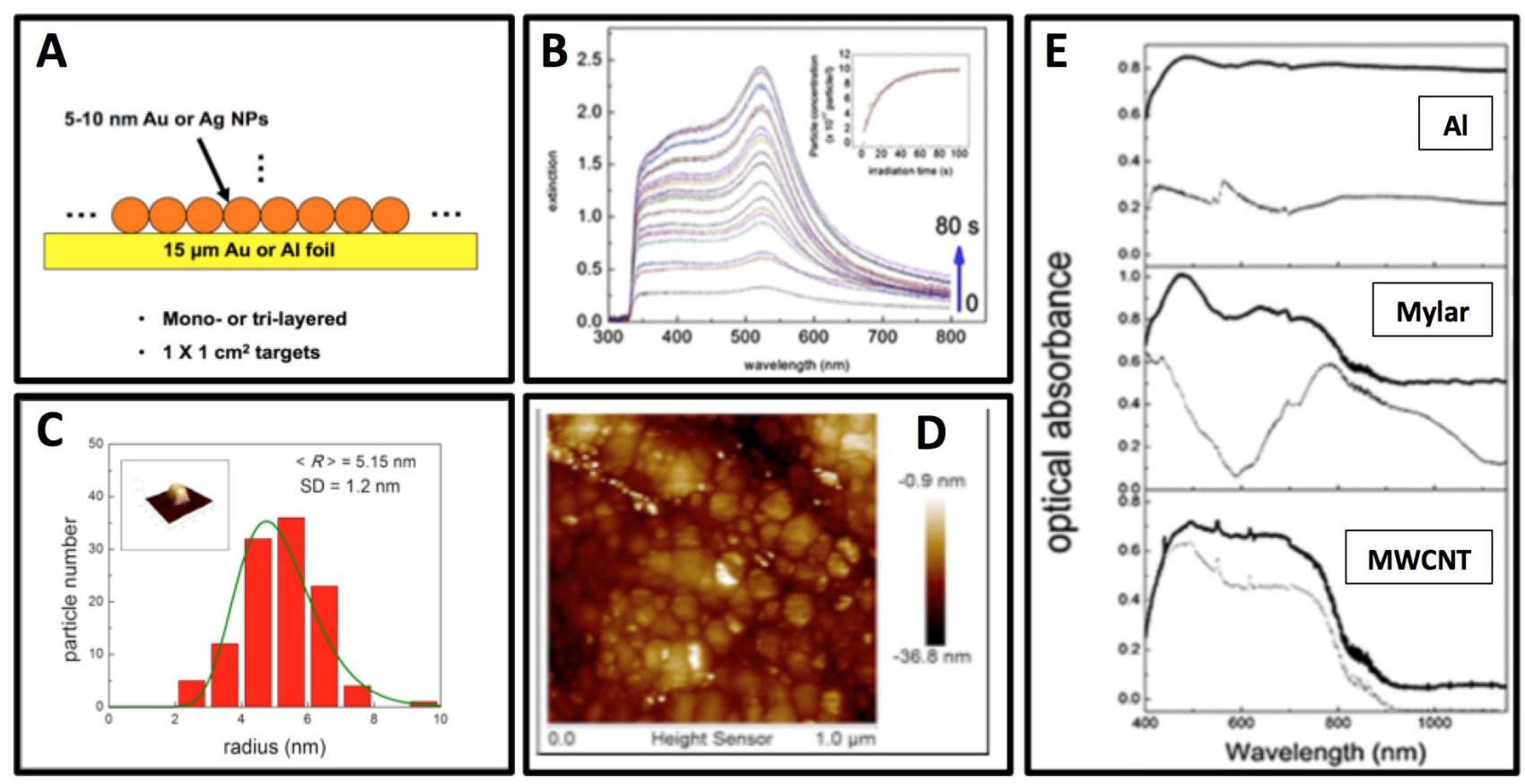

Figure 1. Nanostructured target fabrication (A , B) and characterization (C, D). A) Layer-by-layer deposition of NPs using a controlled spray-dry process. B) Extinction spectrum of GNPs in solution for different ablation times during LASiS. Best case is for 15 seconds of ablation. The inset corresponds to NP concentration in solution with ablation time. C) Nanoparticle size characterization for 100 GNPs analyzed from an AFM image. D) Surface topography of nanostructured target's surface (GNPs on Al foil) using AFM. E) UV-Vis spectroscopy on nanostructured (solid black lines) and non-nanostructured (dotted grey lines) targets.

A sketch of the nanostructured targets is shown in Figure 1A, witnessing the simplicity behind the concept. As foils and nanoparticles are easily accessible nowadays, it makes also these targets relatively easy to fabricate. The extinction spectrum of GNPs in solution after ablation process is shown in Figure 1B, for different ablation times with intervals of 4 seconds. The plasmonic absorption peak of GNPs is clearly visible at around $525 \mathrm{~nm}$. Moreover, the central position of the peak does not shift with ablation time, which shows that the process is very stable and that the nanoparticle distribution is uniform with time. From results obtained, we selected for the growth of the nanostructured films the GNPs obtained after an irradiation time of $15 \mathrm{~s}$., for which we are confident of selecting only the primary particles produced by the laser ablation, excluding big and amorphous clusters which aggregates for greater times. A histogram of the GNP distribution is shown on Figure 1C. Moreover on Figure 1D, an AFM image of GNPs deposited on a 15 microns-thick aluminum foil is shown. The roughness obtained on this surface was of $11 \mathrm{~nm}$, which confirms the presence of a monolayer of GNPs. Finally, as is shown on Figure 1E, all the surfaces coated with GNPs (Mylar, MWCNT and Al) exhibited an increased amount of extinction compared to uncoated foils. The optical absorption of nanostructured materials is characterized by a broad structure covering all the visible range, with 5 broad adsorption peaks at 485, 574, 635, 691, and $776 \mathrm{~nm}$ that are due to the adsorbed GNPs on the surface.

\section{EXPERIMENT ON TITAN LASER}

A laser-driven proton acceleration experiment under TNSA conditions was performed at the Jupiter Laser Facility (JLF) of the Lawrence Livermore National Laboratory (LLNL). We used the TITAN laser which has a central wavelength of $1053 \mu \mathrm{m}$ and a spot size of roughly $10 \mu \mathrm{m}$. Each shot had a pulse energy of $230 \mathrm{~J}$ delivered over 
$700 \mathrm{fs}$, which provides an intensity on the order of $10^{20} \mathrm{~W} / \mathrm{cm}^{2}$. A sketch of the experimental setup is shown on Figure $2 \mathrm{~A}$. An $f / 3$ off-axis parabola was used to focus the laser beam onto $15 \mu \mathrm{m}$-thick aluminum and gold (non-nanostructured and nanostructured) foils as proton generation targets. The protons then pass through a slit before entering into a Thomson parabola to deflect proton trajectories and thus split them in energy. The proton trace was recorded using Image Plates which were analyzed using a homemade software to extract the proton spectra. A typical proton trace is shown on Figure 2B, on which we can clearly see the slit (background X-ray signal passing through the opening) and the proton trace extending to its right side. It is important to note that the distance from the slit $x\left(r_{0}\right)$ is greater with decreasing proton energy $E$, as shown by equation (1), which is derived from proton kinematics' differential equations under the influence of a magnetic field.
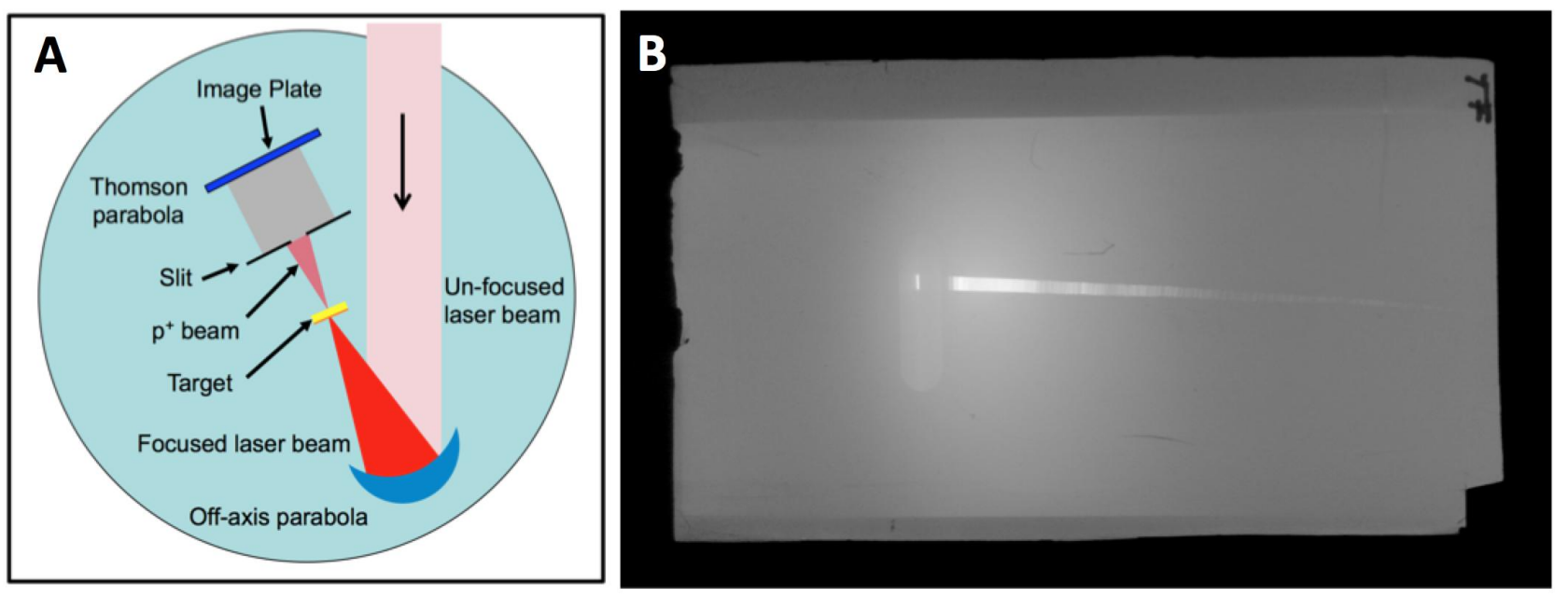

Figure 2. A) Sketch of experimental setup with TITAN laser at LLNL. B) Photostimulated luminescence image of proton trace on Image Plate. One can clearly see the slit position (undeflected trajectory). Proton energy decreases with increasing distance from the slit.

$$
x\left(r_{0}\right)=r_{0}\left\{\cos \left[\arcsin \left(L_{1} / r_{0}\right)\right]-1\right\}-L_{2} \tan \left[\arcsin \left(L_{1} / r_{0}\right)\right],
$$

In equation (1), $r_{0}=\frac{\sqrt{2 E m}}{e B}$ is the curvature radius of the particle's circular trajectory, $m$ is the particle mass, $e$ is the elementary charge and $B$ is the applied magnetic flux density. Moreover, $L_{1}$ is the magnet length and $L_{2}$ is the distance between the magnet and the detection screen, an Image Plate in this case. The Thomson parabola was preliminarily calibrated in order to properly determine the experimental values of $L_{1}, L_{2}$ and $B$ through a fit of calibration data points on equation (1). Once this is done, it is then possible to recover the proton spectra by analyzing images obtained after scanning the Image Plates. Preliminary results of proton spectra obtained from conventional (non-nanostructured) $\mathrm{Al}$ and $\mathrm{Au}$ targets are shown on Figure 3. Proton spectra generated from nanostructured targets are still under analysis and results will be presented in an upcoming publication. We can first note in Figure 3 that spectra for gold and aluminum targets are very similar, although gold targets tend to generally offer a higher cutoff energy due to its greater absorption of the incoming laser energy. The reported cutoff energies are of $52 \mathrm{MeV}$ for $\mathrm{Al}$ and $55 \mathrm{MeV}$ for $\mathrm{Au}$, which are very consistent with the cutoff energies reported in Flippo et al., ${ }^{18}$ where they use a laser of similar scale. Moreover, the fluence scale is also very comparable.

\section{CONCLUSION}

Enhancement of laser-to-particle energy transfer is a crucial parameter for the improvement of laser-driven proton beams. We have investigated the development of nanostructured targets through an easy-to-use and cheap methodology compared to conventional lithographic methods. Gold nanoparticles of $10 \mathrm{~nm}$ in diameter 


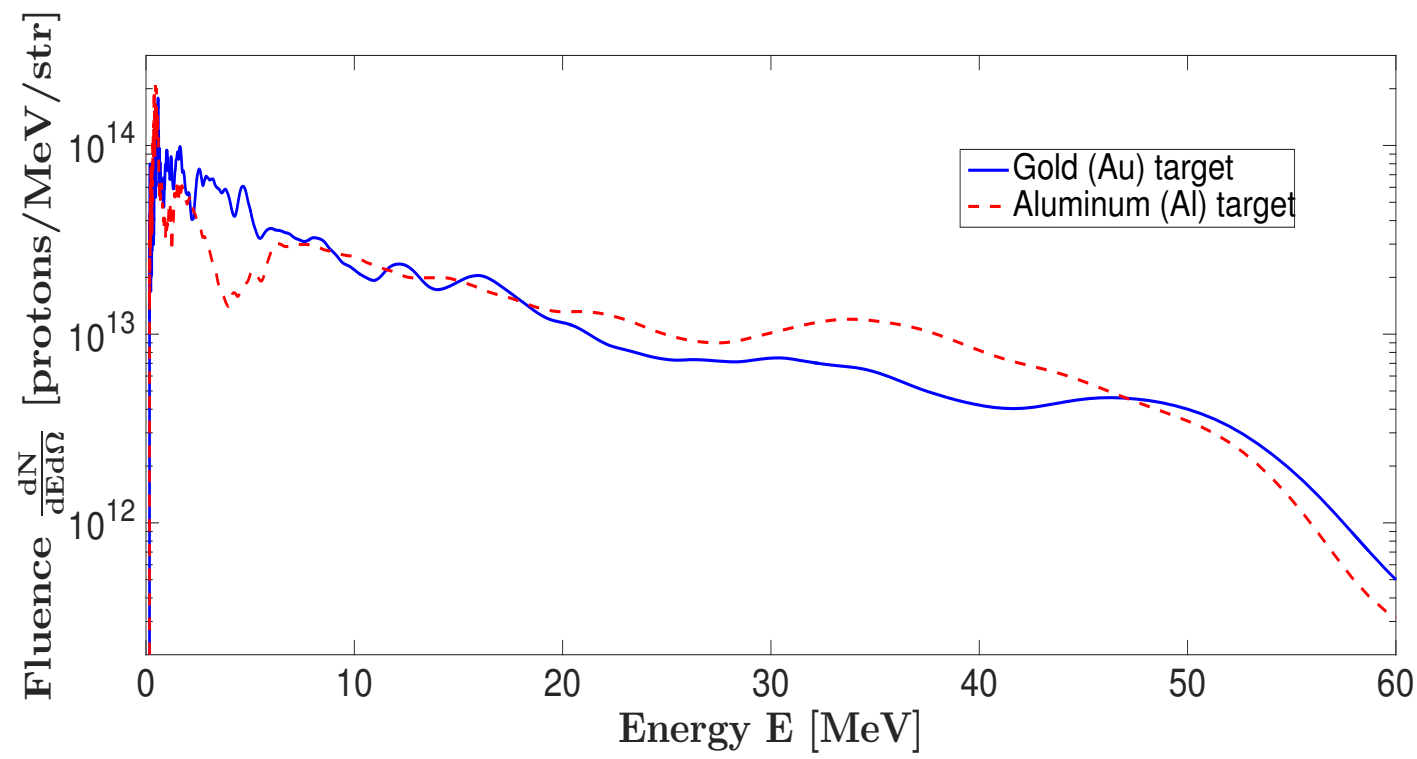

Figure 3. Typical proton spectra obtained with $15 \mu \mathrm{m}$ gold target (blue) and $15 \mu \mathrm{m}$ aluminum target (red) using $230 \mathrm{~J}$ per shot with a pulsewidth of $700 \mathrm{fs}$. Energy cutoff is of around $52 \mathrm{MeV}$ for $\mathrm{Al}$ and $55 \mathrm{MeV}$ for Au, similar to what is reported at Trident, a laser of comparable scale in terms of energy per pulse and pulse. ${ }^{18}$

are produced by laser ablation and then, using a spray-dry process, deposited on aluminum, mylar and MWCNT buckypaper. The increased photon absorption property of the nanostructured films, coming essentially from broadband plasmonic excitations, has been demonstrated through UV-Vis optical spectroscopy. We have then performed an experiment on the TITAN laser at LLNL to demonstrate that nanostructured films are indeed able to increase the laser-to-target absorption, and thus ultimately the laser-to-particle energy transfer. The data concerning proton spectra using nanostructured target is still to be analyzed properly, for which the data will appear in another publication. We will compare nanostructured and non-nanostructured targets' performance through ratios of the proton total fluence, total energy fluence and cutoff energy as metrics to evaluate the enhancement. An important fact to note is that we are not expecting to have an increased laser-to-target absorption that is due to plasmonic oscillations during TNSA experiments, as the pre-pulse of the beam may have already destroyed the nanoparticle layer on the target before the main pulse. However, we are expecting the produced pre-plasma to favor the absorption through an increased amount of collisional and resonant processes. Further experiments are also planned on the LULI laser from École Polytechnique de Paris and as well on the ALLS laser facility at INRS-EMT in order to validate our findings from the experiment on the TITAN laser.

\section{ACKNOWLEDGMENTS}

This work is supported by FRQNT (nouveaux chercheurs, Grant No. 174726, Equipe 2016-PR-189974), NSERC Discovery Grant (Grant No. 435416) as well as the Canada Foundation for Innovation (CFI). Particle-In-Cell simulations ${ }^{19}$ under development are also possible with the use of High Performance Computing resources of ComputeCanada (Job: pve-323-ac, P. Antici). 


\section{REFERENCES}

[1] Shi G. \& Kioupakis E., "Electronic and Optical Properties of Nanoporous Silicon for Solar-Cell Applications", ACS Photonics, vol. 2 (2), 208-2015 (2015).

[2] Aydin K., Ferry V. E., Briggs R. M. \& Atwater H. A., "Broadband Polarization-Independent Resonant Light Absorption Using Ultrathin Plasmonic Super Absorbers", Nat. Commun., vol. 2, 517 (2011).

[3] Rosei F., "Nanostructured Surfaces: Challenges and Frontiers in Nanotechnology", Condens. Mat., vol. 16 (17), S1373 (2004).

[4] Paz V. F. et al., "Development of Functional sub-100 nm Structures with 3D Two-Photon Polymerization Technique and Optical Methods for Characterization", Journal of Laser Applications, vol. 24, 042004 (2016).

[5] Chen A., Komura M., Kamata K. \& Iyoda T., "Highly Ordered Arrays of Mesoporous Silica Nanorods with Tunable Aspect Ratios from Block Copolymer Thin Films", Adv. Mater., vol. 20 (4), 763-767 (2008).

[6] Goda T. \& Iyoda T., "Ultrahigh Density Electrolytic Nanoreactors Composed of Liquid Crystalline Block Copolymer Template: Water-Electrolysis-Induced Deposition of Cerium Oxyhydroxide Nanorod Array", $J$. Mater. Chem., vol. 22, 9477-9480 (2012).

[7] Tong H., Ouyang S., Bi Y., Umezawa N., Oshikiri M., Ye J., "Nano-Photocatalytic Materials: Possibilities and Challenges.", Adv. Mater., vol. 24 (2), 229 (2012).

[8] Wilks S. C., Kruer W. L., Tabak M., \& Langdon A. B., "Absorption of Ultra-Intense Laser Pulses", Phys. Rev. Lett., vol. 69, 1383 (1992).

[9] Schwoerer H. , Pfotenhauer S., Jckel O., Amthor K.-U., Liesfeld B., Ziegler W., Sauerbrey R., Ledingham K. W. D. \& Esirkepov T., "Laser-Plasma Acceleration of Quasi-Monoenergetic Protons from Microstructured Targets", Nature, vol. 439, 445-448 (2006).

[10] Cutroneo M., Torrisi L., Calcagno L. \& Torrisi A., "Characterization of Thin Films for TNSA Laser Irradiation", Journal of Physics: Conference series, vol. 508 (1), 012012 (2014).

[11] Antici P. et al., "Hot and Cold Electron Dynamics Following High-Intensity Laser Matter Interaction", Phys. Rev. Lett., vol. 101, 105004 (2008).

[12] Bagchi S. et al., "Fast Ion Beams from Intense, Femtosecond Laser Irradiated Nanostructured Surfaces", Appl. Phys., vol. 88, 167-173 (2007).

[13] Andreev A. et al., "Efficient Generation of Fast Ions from Surface Modulated Nanostructure Targets Irradiated by High Intensity Short-Pulse Lasers", Phys. Plasmas, vol. 18, 103103 (2011).

[14] Yu J. et al., "Laser-Driven Proton Acceleration Using a Conical Nanobrush Target", Phys. Plasmas, vol. 19, 053108 (2012).

[15] Margarone D. et al., "Laser-Driven Proton Acceleration Enhancement by Nanostructured Foils", Phys. Rev. Lett., vol. 109, 234801 (2012).

[16] Barberio M. et al., "Fabrication of Nanostructured Targets for Improved Laser-Driven Proton Acceleration", Superlatt. \& Microstruc., vol. 95, (2016).

[17] Barberio M., Barone P., Xu F. \& Bonanno A., "Silver Nanoparticles Synthesized by Laser Ablation in Acetone: Influence of Ablation Time and Their Reactivity with Oxygen in the Air", J. Chem. Chem. Eng., vol. 7, (2013).

[18] Flippo K. A. et al., "Scaling laws for energetic ions from the commissioning of the new Los Alamos National Laboratory 200 TW Trident laser", Rev. Sci. Instrum., vol. 79, 10E534 (2008).

[19] Sentoku Y. \& Kemp A.J., "Numerical Methods for Particle Simulations at Extreme Densities and Temperatures: Weighted Particles, Relativistic Collisions and Reduced Currents", Journal of Computational Physics, vol. 227 (14), p. 6846-6861 (2008). 